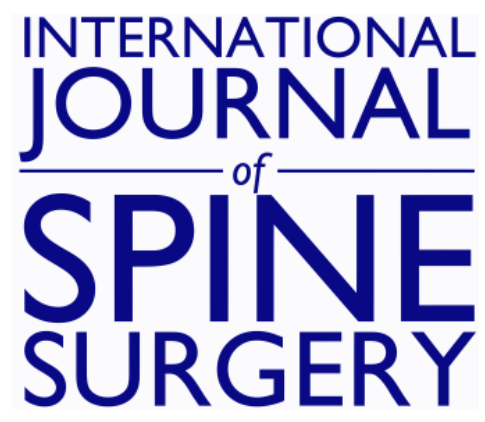

\title{
Robotic assisted surgeries for the treatment of spine tumors
}

Xiaobang Hu, Thomas J. Scharschmidt, Donna D. Ohnmeiss and Isador H. Lieberman

Int J Spine Surg 2015, 9 ()

doi: https://doi.org/10.14444/2001

http://ijssurgery.com/content/9/1

This information is current as of April 26, 2023.

Email Alerts Receive free email-alerts when new articles cite this article. Sign up at: http://ijssurgery.com/alerts 


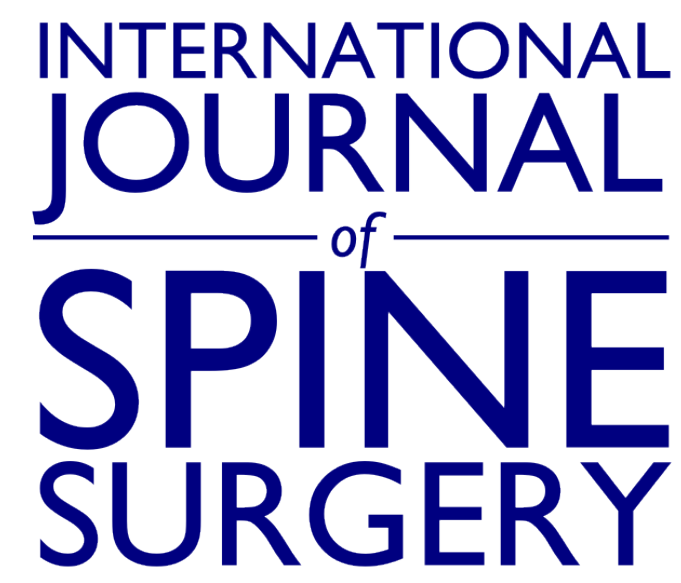

This article generously published free of charge by the International Society for the Advancement of Spine Surgery.

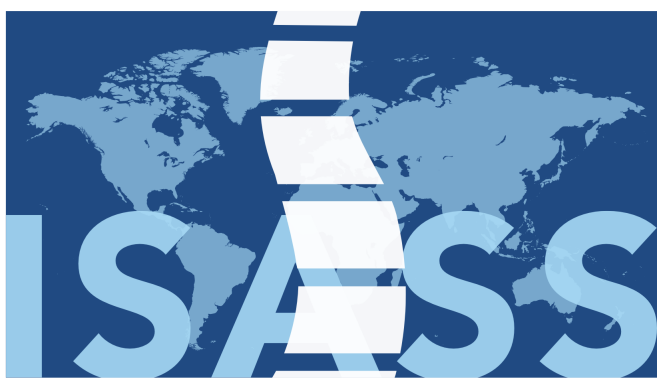

INTERNATIONAL SOCIETY for the ADVANCEMENT of SPINE SURGERY 


\section{Robotic assisted surgeries for the treatment of spine tumors}

Xiaobang Hu, MD, PhD, ${ }^{1}$ Thomas J. Scharschmidt, MD, ${ }^{2}$ Donna D. Ohnmeiss, Dr.Med, ${ }^{3}$ Isador H. Lieberman, $M D, M B A, F R C S C^{l}$

${ }^{1}$ Scoliosis and Spine Tumor Center, Texas Back Institute, Texas Health Presbyterian Hospital Plano, Plano, $T X^{2}$ Department of Orthpaedic Surgery, Wexner medical center/James Cancer Hospital, The Ohio State University, Columbus, OH ${ }^{3}$ Texas Back Institute Research Foundation, Plano, TX

\section{Abstract}

\section{Background}

Surgery plays an important role in the treatment of patients with metastatic or primary spine tumors. In recent years, various new techniques, such as robotic assisted spine surgery have been developed which has shown some promising results by improving the accuracy of spinal instrumentation and reducing potential complications. The purpose of this study was to evaluate our early experience using robotic guidance in the treatment of spinal tumors.

\section{Methods}

Data were collected from medical records for each surgery in which the robotic system was used to assist with biopsy, pedicle screw placement and/or vertebral augmentation in the treatment of spinal tumors. Patient's age, gender, diagnosis and surgical procedure were documented. The surgical time, estimated blood loss, peri-operative and postoperative complications were obtained. The visual analog scale (VAS) for back pain and leg pain were also recorded.

\section{Results}

A total of 9 consecutive patients ( 7 female, 2 male) were included in this study, beginning with the first case experience. The mean age of the patients was 60 years (range 47-69). All patients presented with thoracic or lumbar vertebral collapse and/or myelopathy. Robotic assisted posterior instrumentation was successfully performed in all patients. Robotic assisted vertebral augmentation was performed in 4 patients. The average number of levels instrumented was 5 . The average surgery time (skin to skin) was 4 hours and 24 minutes and the mean blood loss was $319 \mathrm{ml}$. There were no complications perioperatively or through the latest follow-up. Seven of the 9 patients reported improved back pain and/or leg pain at the latest follow-up and the data were not available in two patients. 


\section{Conclusions}

The published complication rates of spinal tumor surgeries range between 5.3\% and $19 \%$. With robotic assistance, the surgical complication rate appears improved over the historical figures. Our study shows that the robotic system was safe and performed as desired in the treatment of metastatic and primary spine tumors. These results support that further evaluation in a larger series of patients.

keywords: Spine tumor, instrumentation, augmentation, robotic-assisted

Volume 9 Article 1 doi: 10.14444/2001

\section{Introduction}

Metastatic spine tumors may occur in up to $30 \%$ of all cancer patients at some point in their disease course. ${ }^{1}$ Spinal cord compression and myelopathy could occur in $25-50 \%$ of these patients. ${ }^{2}$ In contrast, primary spinal cord tumors are rare and only account for $2-4 \%$ of primary central nervous system tumors. ${ }^{3}$ The treatment goals for metastatic and primary spine tumors usually include: to improve patient's quality of life, relieve pain, and maintain or improve neurological function. The most common treatments for metastatic spine tumors are palliative in nature and consist of radiation therapy, systemic chemotherapy, surgery, or some combination of these depending on the histology and behavior of the malignancy. Although non-surgical treatment (radiation and/or chemotherapy) is considered as the first line option for spinal metastasis, surgical intervention has the advantage of rapid and direct decompression of neural structures and immediate stabilization of the spine. The general indications for surgery are spinal instability, progressive symptomatic deformities, neurological deficits, and intractable pain resistant to other treatments. ${ }^{4}$

In recent years, various new techniques, such as computer-navigated and robotic- assisted spine surgery have been developed with the aim to improve the accuracy and consistency of spinal instrumentation placement. ${ }^{5-9}$ Computer-assisted robotic devices are now available in the form of a bone mounted positioning tool that guides the surgeon in the placement of spinal instrumentation according to a pre-operatively planned trajectory (Renaissance, Mazor Robotics Ltd., Israel). ${ }^{5,8}$ This new technology has shown some promising results by increasing the accuracy of spinal instrumentation and reducing potential complications. ${ }^{10-14}$ This technique may be helpful in cases where anatomy is distorted, which is common with tumor destruction of the bone. The purpose of this study was to review our experience in 9 consecutive spine tumor surgeries performed using robotic assisted pedicle screw placement and/or vertebral augmentation. 


\section{Materials and Methods}

\section{Patient Selection}

We conducted a retrospective review of 9 consecutive patients who had robotic assisted pedicle screw placement for spine tumors between December 2010 and December 2013. Patient's age, gender, body mass index (BMI), diagnosis and the history of previous spine surgery were documented. Operative route, level(s) operated, operative time, estimated blood loss, intra-operative and postoperative complications were obtained from medical records. All cases were performed at the same hospital by a single surgeon.

\section{Robotic guidance system and surgical techniques}

The robotic guidance system consists of a cylindrical device with detachable arms that can move in six degrees-of-freedom and a workstation that runs an interface software which facilitates preoperative planning, intra-operative image acquisition and registration, kinematic calculations and real-time robot motion control. In preparation for surgery, a standard CT scan with $1 \mathrm{~mm}$ slices was performed and uploaded to the robotic system planning software. Preoperatively, the surgeon simulated different implants by selecting length, diameter, orientation and the angle of insertion, on a virtual 3-D model of the spine (Figure 1C). If needed, the surgeon simulated the needle trajectories for the cement augmentation component of the procedure. At the start of the operation, the surgeon uploaded the pre-operative plan onto the workstation connected both to the robotic guidance device and to the operating room C-Arm. 

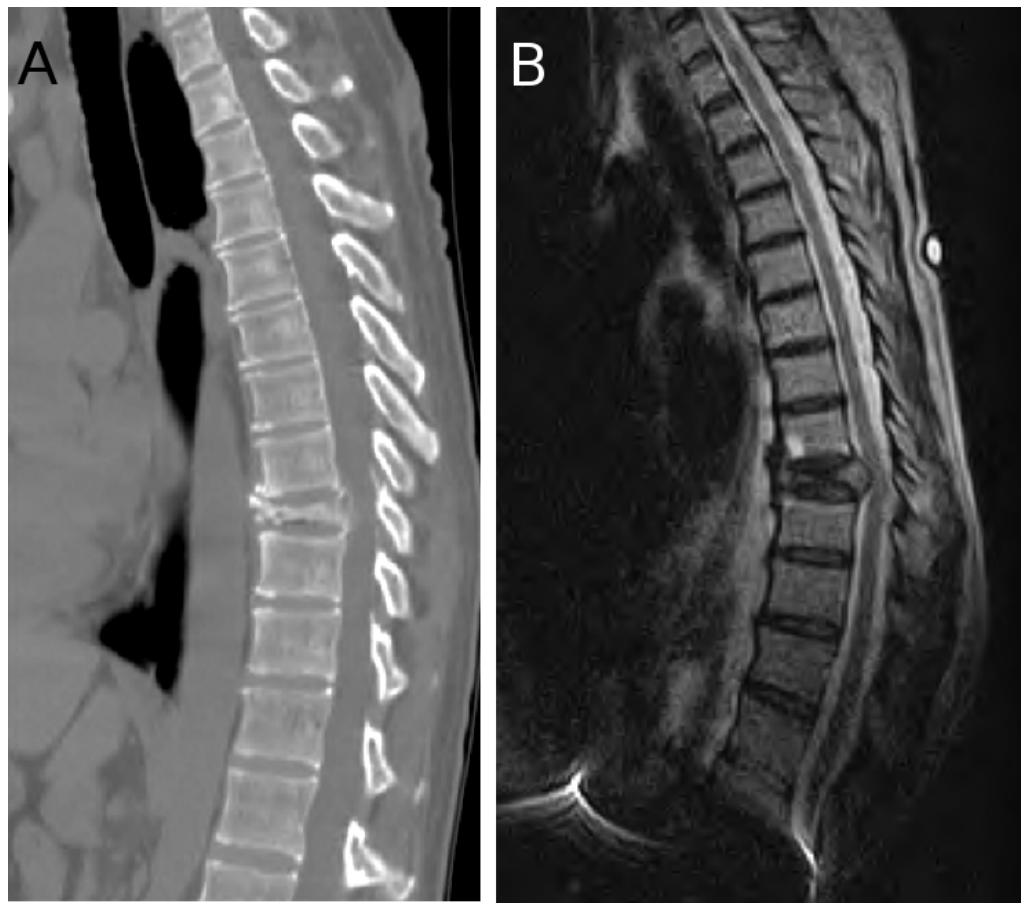

Fig. 1A \& 1B. CT and MRI showing the T9 osteolytic collapse secondary to lung cancer in a 56 year old female (Case 1 ).

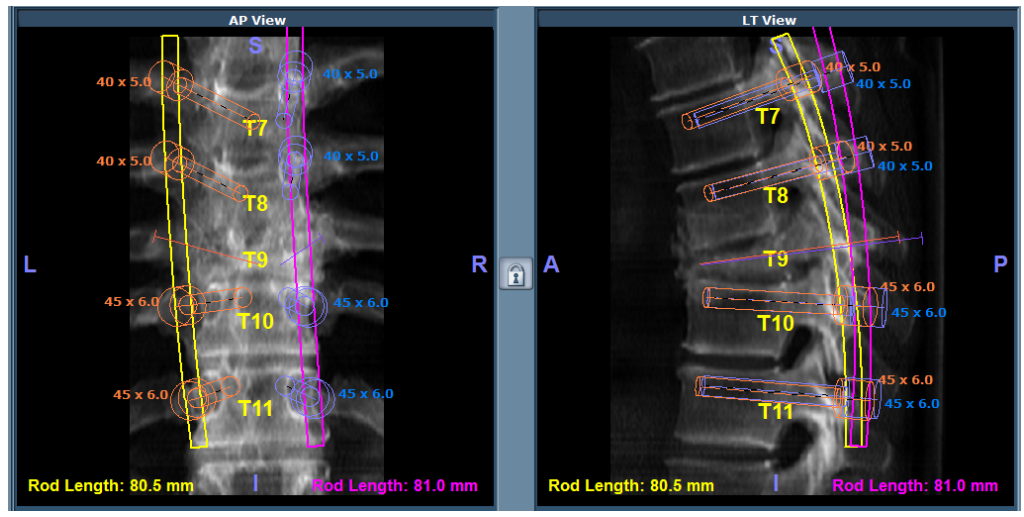

Fig. 1C. Preoperative planning of the robotic assisted pedicle screw placement and vertebral augmentation. 


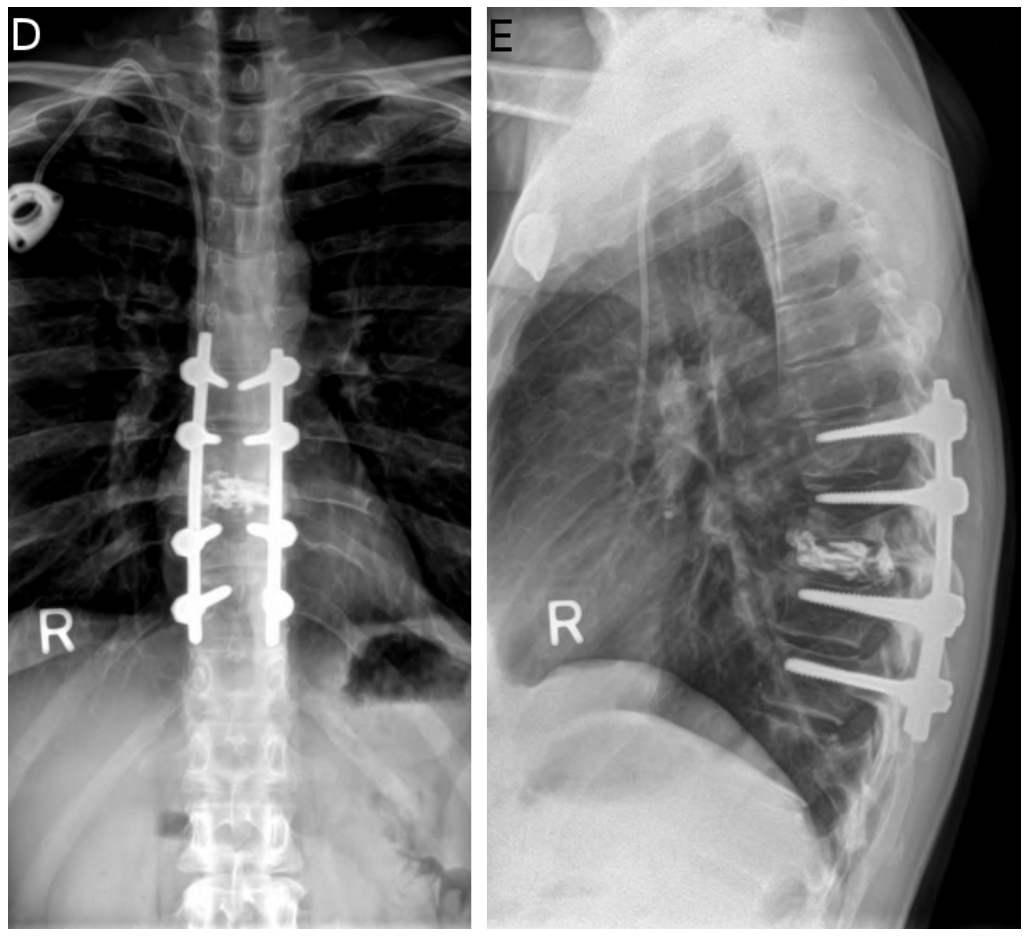

Fig. 1D \&1E. Postoperative $\mathrm{x}$-rays showing that the construct is in good position.

Intraoperatively, once the surgical site was exposed and the levels were verified, the robotic system clamp was attached to the spinous process and the reference frame was placed onto the clamp. An oblique and an AP x-ray were then taken for registration. Then the robotic system bridge was mounted onto the clamp and the robot was mounted onto the bridge. The robot was then dispatched to each one of the stations, to facilitate the trajectory for each pedicle screw and/or the cement augmentation needles, based on the pre-operative plan. Pedicle screws were then implanted and the position of the screws was verified with $\mathrm{x}$-rays.

For vertebral augmentation, $2 \mathrm{~K}$-wires were placed down through the osteolytic pedicles utilizing the robot guidance according to the pre-operative plan. Once placed, the bridge and clamp were removed and a standard vertebral augmentation approach was used to deposit mixed methyl methacrylate into the vertebral body. Spinal decompression and tumor resection procedures were performed as needed.

\section{Outcome measurements}

Visual analog scales (VAS) for back pain, leg pain, neck pain, and arm pain were obtained from each patient preoperatively and at the latest follow-up visit. Pain intensity was measured using a scale ranging from 0 (no pain) to 10 (worst possible pain).

\section{Results}

A total of 9 consecutive patients ( 7 female, 2 male) were included in this study. The mean age of the patients was 60 years (range 47-69). The primary cancer was lung cancer (2 cases), renal cancer ( 2 cases), breast cancer ( 1 case), T12 sarcoma ( 1 case), multiple 
myeloma ( 1 case), adenocarcinoma with unknown origin (1 case), and malignant spindle cell neoplasm with epithelial differentiation and unknown origin (1 case). All patients presented with thoracic or lumbar vertebral collapse and/or myelopathy.

Robotic assisted posterior instrumentation was successfully performed in all patients. Robotic assisted vertebral augmentation was performed in 4 patients (Figure 1). The average number of levels instrumented was 5 (range 3-8). The average surgery time (skin to skin) was 4 hours and 24 minutes (range 1.5-6.5 hours) and the mean blood loss was $319 \mathrm{ml}$ (range 75-1,000 ml). There were no complications peri-operatively or throughout the most recent follow-up. Seven of the 9 patients reported improved back pain and/or leg pain at the latest follow up (between 2 weeks and 1 year postoperatively) and the data were not available in the two patients (Table 1).

Table 1. Patient reported outcome at the latest follow up. NA: not available.

\begin{tabular}{|c|c|c|c|c|c|c|c|c|}
\hline \multirow{2}{*}{$\begin{array}{l}\text { Case } \\
\text { no. }\end{array}$} & \multirow[t]{2}{*}{ Gender } & \multirow{2}{*}{$\begin{array}{l}\text { Age } \\
\text { (years) }\end{array}$} & \multirow{2}{*}{$\begin{array}{l}\text { Site } \\
\text { of Lesion }\end{array}$} & \multirow{2}{*}{$\begin{array}{l}\text { Follow-up } \\
\text { (months) }\end{array}$} & \multicolumn{2}{|c|}{ Back Pain (VAS) } & \multicolumn{2}{|c|}{ Leg Pain (VAS) } \\
\hline & & & & & Preoperative & Postoperative & Preoperative & Postoperative \\
\hline 1 & Female & 56 & T9 & 12 & 6.0 & 0.0 & 0.0 & 0.0 \\
\hline 2 & Female & 57 & L4, L5 & 1 & 9.1 & 0.9 & 8.8 & 0.9 \\
\hline 3 & Female & 67 & $\mathrm{~T} 12$ & 0.5 & 7.3 & 2.5 & 0.0 & 0.0 \\
\hline 4 & Male & 69 & T9 & 3 & 8.0 & 4.0 & 0.0 & 0.0 \\
\hline 5 & Female & 47 & T8, L1, L3 & 1,5 & 8.0 & 5.0 & NA & 4.6 \\
\hline 6 & Male & 69 & T12 & 3 & 0.4 & 0.0 & 0.0 & 0.0 \\
\hline 7 & Female & 58 & L3, L4, L5 & 2 & 10.0 & 2.0 & 10.0 & 0.0 \\
\hline 8 & Female & 49 & $\mathrm{~T} 4, \mathrm{~T} 5$ & NA & NA & NA & NA & NA \\
\hline 9 & Female & 65 & $\mathrm{~T} 12$ & NA & NA & NA & NA & NA \\
\hline
\end{tabular}

\section{Discussion}

Over the last several decades, multimodality treatments such as radiation therapy and chemotherapy have been implemented in the treatment of patients with spine tumors. However, surgery still plays an important role in the treatment of patients with spinal cord compression, instability, or tumors resistant to other modalities. The treatment goals of spinal tumors include the restoration and preservation of neurologic function, provide spinal stability, pain relief and local tumor control. Neurologic deficits in spinal metastases could be caused by the direct anterior tumor compression of the cord, or by posterior extrusion of bone and tumor material. Decompression of the spine can prevent further compression, and allows more rapid rehabilitation.

When metastatic tumors invade the vertebral body and posterior elements, the vertebrae's ability to withstand mechanical loads is usually comprised which will lead to spinal instability. Typical symptoms of spinal instability include pain with movement or axial loading and pain relief with recumbency. For Case 1 in this series, the patient's back pain VAS was 2 when she initially visited our office and surgery was scheduled to decompress 
and stabilize her spine. However, the patient's back pain worsened dramatically soon after and her reported back pain VAS was 6 when she presented to the ER. Once the spinal cord was decompressed and the spine was stabilized the patient's symptoms improved markedly. At 12 months postoperative follow up, she reported no back pain at all.

The role of surgery and benefits achieved in properly selected cases has been well documented in the literature. Patchell and colleagues conducted a prospective randomized clinical trial comparing the outcome of surgery followed by radiotherapy or radiotherapy alone for spinal cord compression caused by metastatic cancer. They decided to terminate the trial as the interim data analysis clearly showed that the patients in the surgical group had superior results when compared to the radiation cohort. Patients treated with surgery also retained the ability to walk significantly longer than those treated with radiotherapy alone and significantly more patients in the surgery group regained the ability to walk than patients in the radiation group. The need for corticosteroids and opioid analgesics was also significantly reduced in the surgical group. ${ }^{15}$ In a systemic literature review, Bilsky et al. found that the ambulatory rates ranged from $73 \%$ to $100 \%$ after surgery and $50 \%$ to $100 \%$ patients regained ambulation. ${ }^{16}$ Wang et al. reported their experience for the treatment of spinal metastases with posterolateral transpedicular approach (PTA) and they found that $96 \%$ of the patients experienced postoperative pain improvement and improvement or stabilization of neurological status. ${ }^{17}$

Pedicle screws are the foundation of spinal instrumentation and they have the advantage of affording multidimensional control and providing substantial rigidity to facilitate fusion. These advantages have led to the wide use of pedicle screws in different spinal reconstruction scenarios including metastatic and primary spine tumors. ${ }^{18,19}$ The accuracy of pedicle screw placement depends largely on the patient' anatomic landmarks and the surgeon's experience. Screw malposition may lead to serious vascular and neurologic complications especially when the patient's anatomy is changed such as in metastatic or primary spinal tumors.

In this report, we used a computer-assisted robot to guide the surgeon in the placement of pedicle screws according to a pre-operatively planned trajectory. This new technology has shown some promising results by increasing the accuracy of spinal instrumentation, reducing potential complications and reducing radiation exposure. ${ }^{10-14}$ Sukovich et al. reported their early clinical experience with the system in 14 patients and found that it performed successfully in $93 \%$ of the cases and $96 \%$ of the screws placed were within 1 mm of the planned trajectory. ${ }^{14}$ Pechlivanis et al. evaluated the accuracy of percutaneous pedicle screw placement in the lumbar spine using this robotic system. As analyzed by postoperative CT scans, out of 133 total pedicle screws placed, in the axial plane, 91.7\% screws were placed exactly within the pedicle and $6.8 \%$ screws deviated $<2 \mathrm{~mm}$. In the longitudinal plane, $81.2 \%$ screws were placed exactly within the pedicle and $9.8 \%$ screws deviated $<2 \mathrm{~mm} .{ }^{13}$ In a retrospective multicenter study, Devito et al. reported that in 682 cases in which 3,912 screw placement were planned, 83.6\% (3,271 screws) were fully implanted under robot guidance, while the remaining screws were initiated under robotic guidance and manually continued by the surgeon. CT scans were available for 646 screws in 139 cases and they demonstrated that $98.3 \%$ of the screws fell within the safe zone. ${ }^{10}$ Recently, the accuracy of robotic-assisted screw placement was evaluated in a consecutive series of 102 patients. Robotic-guided screw placement was successfully used 
in 95 out of 102 patients. In those 95 patients, 949 screws $(87.5 \%$ of 1,085 planned screws) were successfully implanted. Of the 960 screws that were implanted using the robot, 949 (98.9\%) were successfully and accurately implanted and $11(1.1 \%)$ were malpositioned, despite the fact that the majority of patients had significant spinal deformities and/or previous spine surgeries. ${ }^{20}$ Kantelhardt et al. studied the functional outcome of robotic assisted spine surgery and they found that patients operated with robotic assistance required less opioids, had a shorter hospitalization and lower rate of adverse events in the perioperative period comparing to the patients that were operated with the conventional technique. These benefits are even greater for those patients who had robotic assisted percutaneous procedures. ${ }^{11}$

As described in this case series report, bone-mounted robotic guidance can facilitate the accurate placement of pedicle screws in patients with spinal tumors. This technology is also useful for non-pedicle-screw procedures such as biopsies and vertebral augmentations (vertebroplasty and kyphoplasty). The robotic navigation offers the potential benefits of precise pre-operative planning for the more suitable entry points and more appropriate trajectories, via a strategic less invasive exposure, and intra-operative execution of the surgical plan. Based on the results of this small series, further studies are warranted to determine if the use of robotic guidance can translate into improved surgical outcome for patients with spinal tumors.

\section{References}

1. Wong DA, Fornasier VL, MacNab I. Spinal metastases: the obvious, the occult, and the impostors. Spine 1990;15:1-4.

2. Rades D, Abrahm JL. The role of radiotherapy for metastatic epidural spinal cord compression. Nat Rev Clin Oncol 2010;7:590-8.

3. Schellinger KA, Propp JM, Villano JL, McCarthy BJ. Descriptive epidemiology of primary spinal cord tumors. J Neurooncol 2008;87:173-9.

4. Yang SB, Cho W, Chang UK. Analysis of prognostic factors relating to postoperative survival in spinal metastases. J Korean Neurosurg Soc 2012;51:127-34.

5. Lieberman IH, Togawa D, Kayanja MM, et al. Bone-mounted miniature robotic guidance for pedicle screw and translaminar facet screw placement: Part I--Technical development and a test case result. Neurosurgery 2006;59:641-50.

6. Merloz P, Troccaz J, Vouaillat H, et al. Fluoroscopy-based navigation system in spine surgery. Proc Inst Mech Eng H 2007;221:813-20.

7. Shoda N, Nakajima S, Seichi A, et al. Computer-assisted anterior spinal surgery for a case of recurrent giant cell tumor. J Orthop Sci 2002;7:392-6.

8. Togawa D, Kayanja MM, Reinhardt MK, et al. Bone-mounted miniature robotic guidance for pedicle screw and translaminar facet screw placement: part 2-Evaluation of system accuracy. Neurosurgery 2007;60:ONS129-39.

9. Wang HC, Yang YL, Lin WC, et al. Computer-assisted pedicle screw placement for thoracolumbar spine fracture with separate spinal reference clamp placement and registration. Surg Neurol 2008;69:597-601.

10. Devito DP, Kaplan L, Dietl R, et al. Clinical acceptance and accuracy assessment of spinal implants guided with SpineAssist surgical robot: retrospective study. Spine 2010;35:2109-15. 
11. Kantelhardt SR, Martinez R, Baerwinkel S, et al. Perioperative course and accuracy of screw positioning in conventional, open robotic-guided and percutaneous roboticguided, pedicle screw placement. Eur Spine J 2011;20:860-8.

12. Lieberman IH, Hardenbrook MA, Wang JC, Guyer RD. Assessment of pedicle screw placement accuracy, procedure time, and radiation exposure using a miniature robotic guidance system. J Spinal Disord Tech 2012;25:241-8.

13. Pechlivanis I, Kiriyanthan G, Engelhardt M, et al. Percutaneous placement of pedicle screws in the lumbar spine using a bone mounted miniature robotic system: first experiences and accuracy of screw placement. Spine 2009;34:392-8.

14. Sukovich W, Brink-Danan S, Hardenbrook M. Miniature robotic guidance for pedicle screw placement in posterior spinal fusion: early clinical experience with the SpineAssist. Int J Med Robot 2006;2:114-22.

15. Patchell RA, Tibbs PA, Regine WF, et al. Direct decompressive surgical resection in the treatment of spinal cord compression caused by metastatic cancer: a randomised trial. Lancet 2005;366:643-8.

16. Bilsky MH, Laufer I, Burch S. Shifting paradigms in the treatment of metastatic spine disease. Spine 2009;34:S101-7.

17. Wang JC, Boland P, Mitra N, et al. Single-stage posterolateral transpedicular approach for resection of epidural metastatic spine tumors involving the vertebral body with circumferential reconstruction: results in 140 patients. Invited submission from the Joint Section Meeting on Disorders of the Spine and Peripheral Nerves, March 2004. J Neurosurg Spine 2004;1:287-98.

18. Gaines RW, Jr. The use of pedicle-screw internal fixation for the operative treatment of spinal disorders. J Bone Joint Surg Am 2000;82-A:1458-76.

19. Tan JW, Shen BH, Du W, et al. Bone cement enhanced pedicle screw fixation combined with vertebroplasty for elderly patients with malignant spinal tumors. Chin Med J (Engl) 2013; 126:2495-8.

20. Hu X, Ohnmeiss DD, Lieberman IH. Robotic-assisted pedicle screw placement: lessons learned from the first 102 patients. Eur Spine J 2013;22:661-6.

\section{Disclosures}

Xiaobang Hu received a travel support for lecturing from Mazor Robotics in 2011. Donna D. Ohnmeiss is employed by a foundation that receives funding to conduct training labs from Mazor Robotics. Isador H. Lieberman receives royalty, intellectual property rights, consulting fee and ownership interest from Mazor Robotics. No funds were received in support of this work.

\section{Corresponding Author}

Isador H. Lieberman, Scoliosis and Spine Tumor Center, Texas Back Institute, Texas Health Presbyterian Hospital Plano, 6020 W. Parker Rd., Ste. 200a, Plano, TX 75093, USA. ilieberman@texasback.com

Published 3 February 2015.

Copyright (C) 2015 ISASS - International Society for the Advancement of Spine Surgery. To see more or order reprints or permissions, see http://ijssurgery.com. 